\title{
Evaluation of the Effect of Input Stimuli on the Quality of Orientation Maps Produced Through Self Organization
}

\author{
A. Ravishankar Rao, Guillermo Cecchi, Charles Peck, and James Kozloski
}

IBM T.J. Watson Research Center, Yorktown Heights, NY 10598, USA

ravirao@us.ibm.com

\begin{abstract}
Self-organized maps have been proposed as a model for the formation of sensory maps in the cerebral cortex. The role of inputs is critical in this process of self-organization. This paper presents a systematic approach to analyzing the relationship between the input ensemble and the quality of self-organization achieved.

We present a method for generating an input stimulus set consisting of images of curved lines. The advantage of this approach is that it allows the user the ability to precisely control the statistics of the input stimuli to visual processing algorithms. Since there is considerable scientific interest in the processing of information in the human visual stream, we specifically address the problem of self-organization of cortical visual areas $\mathrm{V} 1$ and V2.

We show that the statistics of the curves generated with our algorithm match the statistics of natural images. We develop a measure of self-organization based on the oriented energy contained in the afferent weights to each cortical unit in the map. We show that as the curvature of the generated lines increases, this measure of self-organization decreases. Furthermore, self-organization using curved lines as stimuli is achieved much more rapidly, as the curve images do not contain as much higher order structure as natural images do.
\end{abstract}

\section{Introduction}

Several algorithms for the self-organizing formation of orientation columns in the visual cortex have been proposed [3]. The visual cortical area V1 receives retinal input via the thalamus and projects to a higher-level area V2. The role of inputs is critical in the process of self-organization. Hubel et al [5] showed that rather than being genetically predetermined, the structure of cortical visual area V1 undergoes changes depending on the animal's visual experience, especially during the critical period of development. Sharma et al [9] showed that rewiring the retinal output to the auditory cortex instead of the visual cortex resulted in the formation of orientation-selective columns in the auditory cortex. It is thus likely that the same self-organization process is taking place in different areas 
of the cortex. The nature of the cortical maps then becomes a function of the inputs received.

Thus, a study of the input space plays an important role in furthering our understanding of cortical maps. In this paper we investigate the presentation of the proper visual inputs that are necessary to achieve the type of self-organization observed in the visual cortex, including areas V1 and V2 [3].

The choice of input stimuli is related to the desired modelling task. Certain classes of inputs are sufficient to model V1. For instance, Bednar [1] used input stimuli consisting of elongated Gaussian blobs. However, if the goal is to model higher order cortical areas such as V2, this class of stimuli is not appropriate. One of the problems with using Gaussian blobs is that they do not provide sufficient information for the self-organization of higher-order maps such as V2, whose elements have wider receptive fields than the elements of V1.

Other researchers have used natural images [6] as inputs to self-organizing algorithms. However, the bulk of such efforts are aimed at modeling area V1, and no higher.

Since the self-organization of cortical maps is critically dependent on the input ensemble, a thorough and systematic study of the dependence of the quality of self-organization with respect to the inputs is desirable. The goal of this paper is to present algorithms for the generation of images of curved lines, which are then used for testing methods for visual processing, such as cortical simulations of the visual stream, including the organization of V1 and V2. The advantage of this approach is that it allows the user the ability to precisely control the statistics of the input stimuli to visual processing algorithms. We show that the statistics of the curves generated with our algorithm are similar to the statistics of natural images as determined by Sigman et al [10]. This way, the self-organizing maps trained by images of artificially generated curved lines should be comparable to the maps generated by training on natural images, as they are both capturing similar input statistical variations [11].

\section{Background}

Our broader research effort is aimed at modeling interactions between V1 and V2 [8], and in general, the self-organization of higher-order maps in the visual stream. The psychophysical observations of Field et al [4] serve as a testing ground for our model. Field showed that the human visual system is capable of responding to contours of smooth dashed lines in a field of distractors. We are developing a model which provides a neurobiologically grounded explanation for Fields's psychophysical experiment, which involves dashed curved contours. A model that seeks to explain this experiment will only be successful if the appropriate stimuli, namely those containing curved lines, are presented.

Though natural images can be used as input, they must be properly selected to ensure that the right distribution of curved stimuli exists in these images. Furthermore, it is hard to carry out analytics with natural images, as it is difficult to create a mathematical model that precisely describes a natural image. By 
using computer graphics-generated stimuli, we have ready access to a precise mathematical model. A systematic study comparing the properties of computer graphics-generated stimuli and natural images for the purposes of training selforganization algorithms has not been done previously. This paper addresses this issue.

A second problem that has received little attention in the literature is the characterization the sensitivity of the self-organization algorithms to the statistics of the inputs they are trained on. We show that self-organization that parallels cortical area V1 in terms of orientation columns occurs only for certain ranges of curvature in the curved line stimuli. This type of analysis will prove useful to researchers in self-organized maps. Though different researchers have tried several approaches to self-organization with different inputs [3], we propose a systematic approach to explore the dependence of self-organization on the inputs.

\section{$3 \quad$ Methods}

The requirements for the curve-generation algorithm we seek are (1) Generation of realistic curves in terms of their geometry. We use the curvature as a measure of the curve's geometry. (2) The realistic rendering of the curves in terms of their appearance as grayscale images. This implies the use of proper anti-aliasing techniques. (3) The ability to control the spatial distribution of the curves. For instance, it is desirable to ensure that each unit in the cortex has been stimulated an equal number of times by lines of different orientation, such that the distribution of orientations is uniform.

We note that the visual cortex receives retinal inputs via the lateral geniculate nucleus (LGN), whose units perform a center-surround filtering which is similar to edge detection. Thus, the generation of thin contours should suffice to model the output of the LGN to the cortex. The following algorithm is designed to meet the above requirements.

\subsection{Generation of Curve Geometry}

We used an algorithm that manipulates the curvature explicitly, and can be thought of as a random walk in curvature space. The curvature is defined as $\kappa=d s / d \theta$ where $s$ is the arc length and $\theta$ is the tangent angle. The arc length is integrated as we proceed along the curve. The algorithm starts at an initial point $(x, y)$ with tangent angle $\theta$. The curvature is perturbed by a random value, $\eta$, drawn from a uniform distribution in the range $[-0.5,0.5]$. In order to prevent the curvature from changing abruptly, the curve possesses an inertia consisting of the past curvature values. Thus, if the curvature at a time step $t$ is $\kappa(t)$, we maintain the values $\kappa(t-1)$ and $\kappa(t-2)$. The curvature update equation is in the form of a moving-average filter as follows.

$$
\kappa(t+1)=0.5 \kappa(t)+0.3 \kappa(t-1)+0.2 \kappa(t-2)+\gamma \eta
$$


where $\gamma$ is a gain term that controls the amount of noise added. We used $\gamma=0.75$. We would also like to avoid curves with high curvature, as this would result in a spiral behavior of the curve, resulting in rapid termination. This is achieved by imposing a hard limit on the curvature as follows

$$
\kappa(t)=\left\{\begin{array}{l}
T \text { if } \kappa(t)>T \\
-T \text { if } \kappa(t)<-T
\end{array}\right.
$$

The curvature threshold $T$ is determined to be $T=4 / W$ where $W$ is a measure of the size of the image given by the average of its height and width. We make $T$ a function of $W$ so that similar looking curves can be generated for any desired image size.

Next, we compute the new slope, $\mu$ as follows.

$$
\mu(t+1)=\mu(t)+s \kappa(t+1)
$$

where $s$ is the arc-length. For the sake of simplicity, we set $s=1$.

In order to give the appearance of a slowly varying curve, the curvature is updated only every $N$ iterations, say $N=4$.

\subsection{Rendering of the Curve}

Once the geometry of the curve is specified as above, we need to render it in the form of a digital grayscale image. This requires the use of anti-aliasing techniques. We adapt a technique proposed by $\mathrm{Wu}[12]$ to render anti-aliased straight lines. Given a point $(x, y)$ that the curve passes through, with a tangent angle $\theta$, we determine the next digital grid point it must pass through. Let us assume the next point in positive direction of $\theta$ the has an $x$ coordinate of $x(t+1)=$ $x(t)+\Delta x$, without loss of generality. Here, $\Delta x$ is the increment in the $x$ direction and is set to 1 for simplicity. (An analogous procedure is followed to draw the curve in the negative direction of $\theta$ ). $y$ is computed from the slope $\mu$ obtained from equation 3 as

$$
y(t+1)=\mu \Delta x+y(t)
$$

Assuming a grayscale value in the range [0..1] for the image, the next point on the curve, with $x$ coordinate of $x(t)+\Delta x$ is rendered as follows. Let $y_{l}$ be the floor of $y(t+1)$ and $y_{u}$ be the ceiling of $y(t+1)$. The grayscale value $I$ assigned to point $\left(x+\Delta x, y_{l}\right)$ is

$$
I\left(\left(x+\Delta x, y_{l}\right)=y_{u}-y(t+1)\right.
$$

and to point $\left(x+\Delta x, y_{l}\right)$ is

$$
I\left(\left(x+\Delta x, y_{u}\right)=y(t+1)-y_{l}\right.
$$

This results in a thin, anti-aliased curved line, which is an appropriate stimulus to use when testing cortical self-organization algorithms.

We can specify the initial condition that the curve passes through a point $\left(x_{0}, y_{0}\right)$ and has orientation $\theta_{0}$ at this point. The rendering of the curve is performed according to equation 4 in the directions of $+\theta_{0}$ and $-\theta_{0}$. This initial condition is important, as it can be used to ensure that every point in an input image has been visited and every orientation about this point has also been covered. 


\section{$4 \quad$ Experimental Results}

The results of applying the above algorithm for curve generation are shown in Fig. 1. The images are of size 200x200. As can be observed, the resulting curves possess a gradual change in curvature, giving them a snake-like appearance.

The ability of the user to specify the initial conditions for the curve are advantageous in that one can ensure that all points in the image have been visited, and all orientations about each point have been rendered.
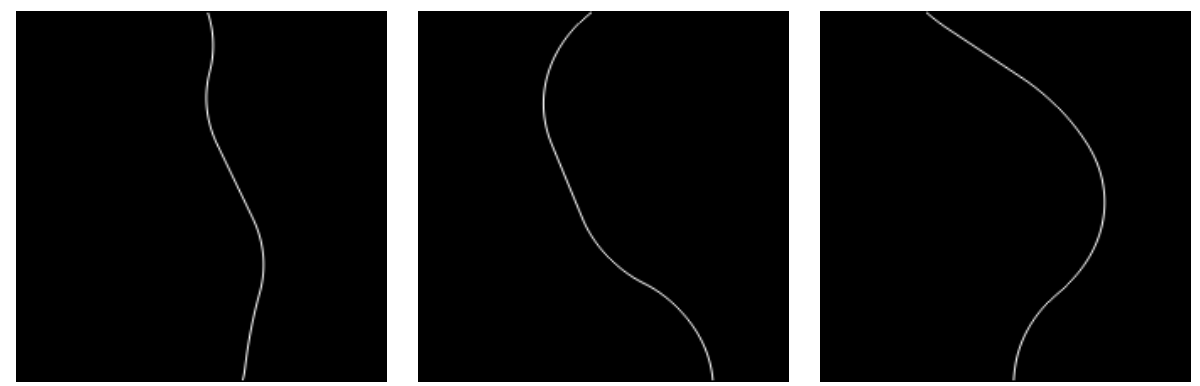

Fig. 1. Sample curves generated by the algorithm

One can vary the rendering of the curve to create dashed lines by using equations 5 and 6 only every $N$ steps.

We now examine the effect of varying the parametrization of the curves on the self-organization process.

\subsection{Effect of the Curve Parameters on Self-organization}

We used the curve generation algorithm described above as input to a selforganization algorithm based on the infomax criterion as developed by Bell and Sejnowski [2]. Consider a network with an input vector $\mathbf{x}$, a weight matrix $\mathbf{W}$, a bias vector $\mathbf{w}_{\mathbf{0}}$, and a squashing function $g$ which creates the output $\mathbf{y}$, where $\mathbf{y}=g\left(\mathbf{W} \mathbf{x}+\mathbf{w}_{\mathbf{0}}\right)$. Let $g$ be a sigmoidal function, with $g(u)=\left(1+e^{-u}\right)^{-1}$. With this form of $g$, the learning rules to achieve mutual information maximization between $\mathbf{x}$ and $\mathbf{y}$ are

$$
\begin{gathered}
\Delta \mathbf{W} \propto\left[\mathbf{W}^{T}\right]^{-1}+(\mathbf{1}-2 \mathbf{y}) \mathbf{x}^{T} \\
\Delta \mathbf{w}_{\mathbf{0}} \propto \mathbf{1}-2 \mathbf{y}
\end{gathered}
$$

We apply the above learning rules to a network with a $10 \mathrm{x} 10$ input layer and a 10x10 output layer, such that each unit in the input layer is connected to every input in the output layer. Let $j$ denote an index into the input layer, and $i$ into the output layer, such that $i, j \in[1,100]$. The weight matrix $\mathbf{W}$ is thus of size $100 \times 100$, where the element $W_{i j}$ describes the connectivity from 
unit $j$ in the input layer to unit $i$ in the output layer. The result of training can be visualized through Fig. 2. The weights that connect to the $i^{\text {th }}$ unit can be arranged in a matrix $\tilde{\mathbf{W}}_{i}$, where the entry $W_{i}(m, n)$ is the weight from an input at location $(m, n)$ to the $i^{\text {th }}$ output unit. In our case, $\tilde{\mathbf{W}}_{i}$ is a $10 \mathrm{x} 10$ matrix. Fig. 2 displays the matrices $\tilde{\mathbf{W}}_{i}$ as graylevel images. Note that several of the weight matrices shown have an oriented structure, showing that they are detecting lines of different orientation, at different phases. There are also some weight matrices that do not show such organization.

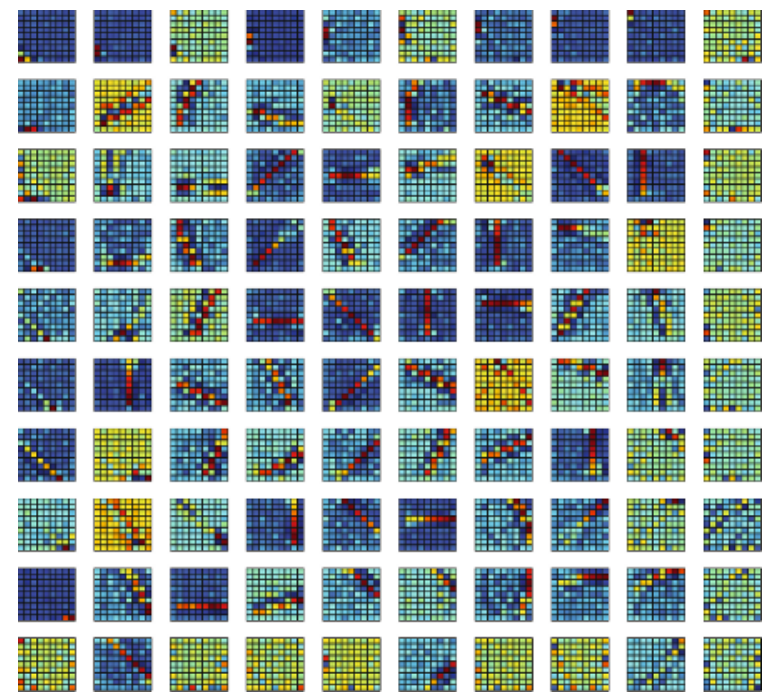

Fig. 2. Result of training using Bell and Sejnowski's algorithm. Each sub-image represents a 10x10 weight matrix for that location in the output layer

In an ideal case, all the weight matrices $\tilde{\mathbf{W}}_{i}$ will have oriented structure after sufficient training. On the other hand, if there is poor self-organization, we will see little oriented-selective structure in the weight matrices. In order to quantify the degree of self-organization ${ }^{1}$, we develop a metric, $d$, based on the amount of oriented energy in a matrix. $d$ depends on a shape measure $s_{i}$ and an energy measure $\eta_{i}$.

In order to define the shape measure $s_{i}$, we threshold the matrix $\tilde{\mathbf{W}}_{i}$ into a binary image using the method of Otsu [7] to yield a region $\mathrm{R}$ of 'ones'. We

\footnotetext{
${ }^{1}$ A more complete measure of self-organization will include the topological organization of the orientation-selective weight matrices. For instance, one could measure the correlation amongst neighboring weight matrices. However, for the sake of simplicity we did not develop such a topological measure, which is a topic for future research.
} 
calculate second order central moments, $\mu_{20}, \mu_{02}$ and $\mu_{11}$ where a central moment of order $p q$ for the region $R$ is defined as

$$
\mu_{p q}=\frac{1}{A} \sum_{x, y \in R}(x-\bar{x})^{p}(y-\bar{y})^{q}
$$

where $A$ is the area of the region $R$ and $(\bar{x}, \bar{y})$ is the centroid of $R$, and $p, q=$ $0,1,2, \ldots$ If the elements of the matrix are considered to be a $2 \mathrm{D}$ image which has an intrinsic shape, then one can define measures of this shape. The shape measure we use, $s_{i}$ is defined by

$$
s_{i}=\frac{\sqrt{\left(\mu_{20}-\mu_{02}\right)^{2}+4 \mu_{11}^{2}}}{\mu_{20}+\mu_{02}}
$$

The shape measure $s_{i}$ reflects the eccentricity of a region, and varies between 0 for a circle and 1 for a highly-elongated region.

Next, consider the mean squared energy $\eta_{i}$ defined by

$$
\eta_{i}=\sum_{m=1}^{m=N} \sum_{n=1}^{n=N} \tilde{\mathbf{W}}_{i}(m, n)^{2} / N^{2}
$$

The measure of oriented energy is then defined by the following product $d_{i}=$ $\eta_{i} s_{i}$. The reason we use such a measure is because the shape measure $s_{i}$ is scale invariant, and a weight matrix consisting of a single blob of noise can have high eccentricity, giving rise to the false conclusion that the matrix shows good self-organization. The global measure of self-organization, $d$, is defined as the average of the measures $d_{i}$, as $d=\sum d_{i} / M$, where $M$ is the number of matrices considered, 100 in this case.

\subsection{Variation of $d$ with the Number of Training Iterations}

As Fig. 3 shows, the measure $d$ of self-organization increases as the number of training iterations increases. The rate of increase in the self-organization is higher in the beginning, and slows down with an increasing number of iterations.

\subsection{Variation of $d$ with Respect to the Curvature Gain}

As Fig. 4(a) shows, the measure $d$ of self-organization decreases with respect to the curvature gain $\gamma$ in equation 1 . This shows that if straight lines are used as stimuli, the map shows better self-organization. However, using straight lines as the sole input form may not result in the correct organization of association fields in V2 [4], which are essential to the line completion experiment described in Section 2. This is because V2 is conjectured to capture information related to curvature of lines [4].

\subsection{Variation of $d$ with the Amount of Noise in the System}

We corrupt each pixel in the the curved line image with additive noise which is drawn from a uniform distribution with zero mean and in the range $[-\delta, \delta]$. 


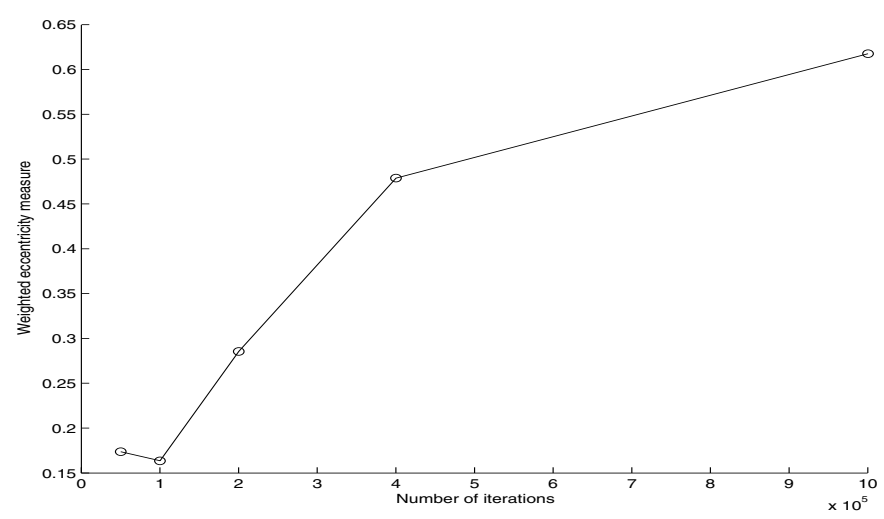

Fig. 3. Plot of the weighted eccentricity measure, $d$ versus the number of iterations
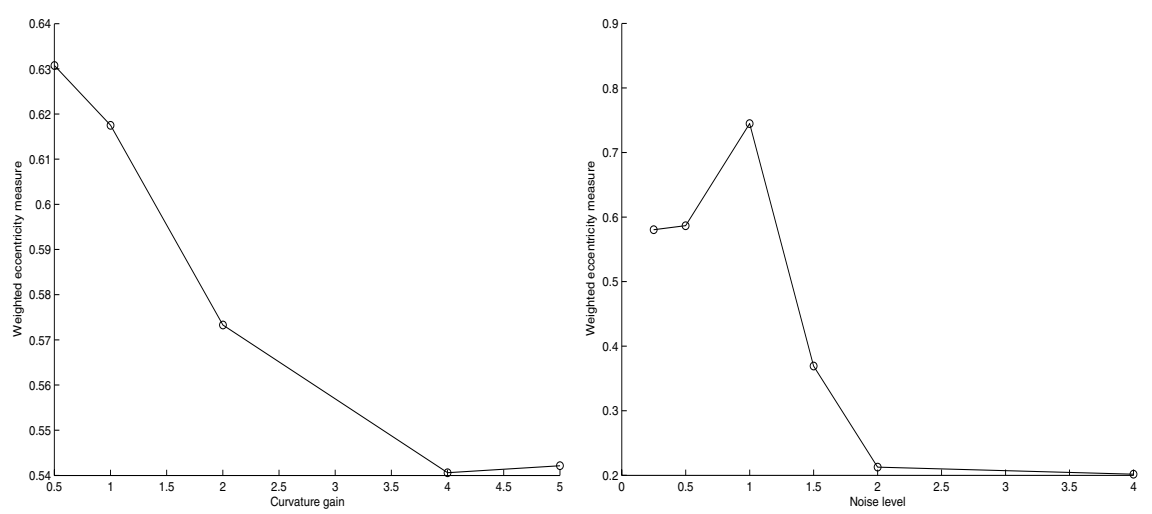

Fig. 4. (a) Plot of the weighted eccentricity measure, $d$ versus the curvature gain, $\gamma$. A fixed training cycle consisting of 1 million iterations was used for all cases. (b) Plot of the weighted eccentricity measure, $d$ versus the noise level, $\delta$. A fixed training cycle consisting of 1 million iterations was used for all cases

Fig. 4(b) shows that the measure $d$ of self-organization decreases with increasing noise level, $\delta$. It is expected that natural images will contain a fair amount of noise. (For instance, the contours of tree branches may be interrupted by foliage). This shows that if synthetic curved lines are used as stimuli, the resulting map shows better self-organization.

Furthermore, to achieve a comparable level of self-organization as measured by $d$, an algorithm using natural images would need to use a much larger number of training cycles, typically by a factor of two times. This can be seen by comparing Fig. 3 and 4(b). 


\subsection{Comparison of the Curve Statistics with That of Natural Images}

We follow the method of Sigman [10] et al to generate statistics of images containing curved lines drawn by our algorithm. Let $E(x, y, \phi)$ denote the energy at pixel $(x, y)$ of an image, where $\phi$ is the dominant orientation at this point. $E(x, y, \phi)$ and $\phi$ are computed using steerable filters as described in [10]. We are interested in the co-occurrence statistics of a line at orientation $\phi$ with a line of orientation $\psi$ at a distance $(\Delta x, \Delta y)$ away. This is expressed as

$$
C(\Delta x, \Delta y, \phi, \psi)=\frac{1}{N} \sum_{n=1}^{N} \iint E_{n}(x, y, \phi) E_{n}(x+\Delta x, y+\Delta y, \psi) d x d y
$$

where $N$ is the total number of images and the integral is calculated for each image. The angles are quantized into 16 equal bins from 0 to $180^{\circ}$. Fig. 5 shows the result of applying this method to a set of 2000 curved lines drawn by the algorithm in Sec. 3.2. Fig. $5(\mathrm{~A})$ shows the co-occurrence matrix $C(\Delta x, \Delta y, 0,0)$, i.e. the co-occurrence of energy for collinear segments. This matrix is displayed as a grayscale 2D image. Fig. 5(B) shows a plot of the logarithm of the distance in pixels on the $x$ axis versus the logarithm of the collinear energy on the $y$ axis. This plot clearly shows that the distribution of collinear energy is not an exponential function, but follows a power law, as anticipated by Sigman et al [10]. Furthermore, collinear interactions extend over long distances.
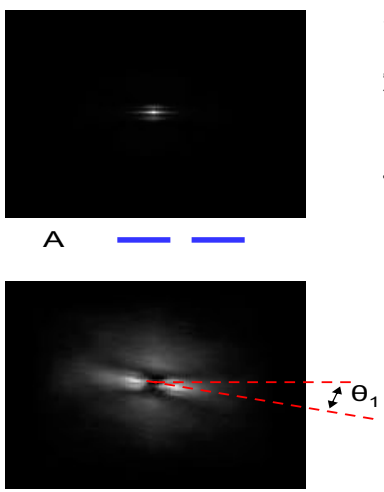

C
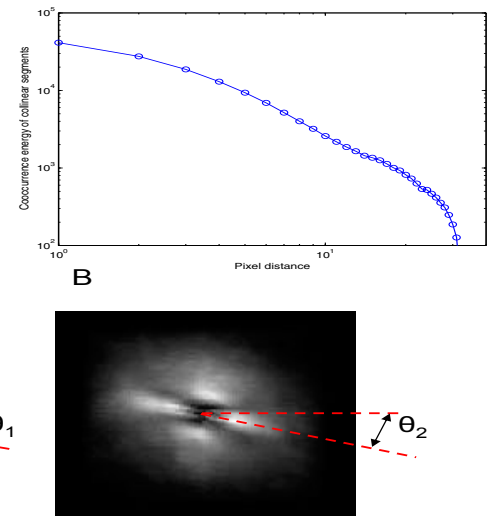

D
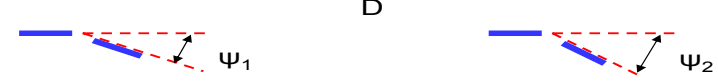

Fig. 5. This figure shows the statistics of energy co-occurrence for various orientations

Fig. 5(C) and (D) explore the phenomenon of co-circularity, which refers to the tendency of pairs of oriented edges in natural images to be co-circular, ie tangent to a common circle [10]. Fig. 5(C) shows $C\left(\Delta x, \Delta y, 0, \psi_{1}=22^{0}\right)$. The 
lobes of maximum intensity have orientation $\theta_{1}=11^{0}$, which is half of $\psi_{1}$, as predicted by the co-circularity rule in Sigman et al [10]. Similarly, Fig. 5(D) shows $C\left(\Delta x, \Delta y, 0, \psi_{2}=34^{0}\right)$. The lobes of maximum intensity in this case have orientation $\theta_{2}=16.5^{0}$, which is approximately half of $\psi_{2}$, as predicted by the co-circularity rule in Sigman et al [10].

The statistics of the artificially generated curved lines as depicted in Fig. 5 are in agreement with those of natural images as shown in Fig. 2 and Fig. 4 of Sigman et al [10] in terms of their power law distribution and co-circularity This shows that using curved lines suffices to train self-organizing maps to achieve organization similar to that in cortical areas V1 and V2.

\section{Conclusions}

In this paper, we developed a systematic approach to analyze the variation of self-organized maps with respect to variations in their input. We presented the benefits of using an artificial curve-generation algorithm for generating inputs to self-organized maps. We developed a measure for the degree of self-organization, and analyzed the behavior of a self-organization method, infomax, with respect to variations in the input. We showed that the amount of curvature of the curved lines affects the degree of self-organization. We also showed that the degree of self-organization decreases with increasing noise in the image.

Thus, it is possible to achieve self-organization in cortical simulations of V1 and V2 faster through the use of the curved line inputs than with images of natural scenes. Since the statistics of the two types of inputs are similar, the fidelity of the maps in representing the statistics of natural scenes is not compromised. The use of the curved line generation algorithm should thus be of great value to researchers who are investigating computational models of the visual cortex.

\section{References}

1. James A. Bednar. Learning to See: Genetic and Environmental Influences on Visual Development. PhD thesis, Department of Computer Sciences, The University of Texas at Austin, 2002. Technical Report AI-TR-02-294.

2. A.J. Bell and T. J. Sejnowski. An information-maximization approach to blind separation and blind deconvolution. Neural Comput., 7(6):1129-1159, 1995.

3. E.Erwin, K. Obermayer, and K. Schulten. Models of orientation and ocular dominance columns in the visual cortex: A critical comparison. Neural Computation, 7(3):425-468, 1995.

4. D. Field, A. Hayes, and R. Hess. Contour integration by the human visual system: Evidence for a local association field. Vision Research, 33(2):173-193, 1993.

5. D.H. Hubel, T.N. Wiesel, and S. Levay. Plasticity of ocular dominance columns in monkey striate cortex. Phil. Trans. R. Soc. Lond. B, 278:377-409, 1977.

6. A. Hyvärinen, P. O. Hoyer, and J. Hurri. Extensions of ICA as models of natural images and visual processing. pages 963-974, Nara, Japan, April 2003.

7. N. Otsu. A threshold selection method from gray-level histograms. IEEE Transactions on Systems Man and Cybernetics, 9(1):62-66, 1979. 
8. A. R. Rao, C. C. Peck, G. A. Cecchi, and J. R. Kozloski. Contour completion and figure ground separation using self-organizing cortical maps. Society for Neuroscience Annual Meeting, October 2004.

9. J. Sharma, A. Angelucci, and M. Sur. Induction of visual orientation modules in auditory cortex. Nature, 404:841 - 847, April 2000.

10. M. Sigman, G. Cecchi, C. Gilbert, and M. Magnasco. On a common circle: natural scenes and gestalt rules. PNAS, 98(4):1935-1940, Feb 2001.

11. E. P. Simoncelli and B. Olshausen. Natural image statistics and neural representation. Annual Review of Neuroscience, 24:1193-1216, March 2001.

12. X. Wu. An efficient antialiasing technique. SIGGRAPH Comput. Graph., 25(4):143-152, 1991. 